\title{
Drinking Motives and Links to Alcohol Use in 13 European Countries
}

\author{
EMMANUEL KUNTSCHE, PH.D., $, a, *$ SAOIRSE NIC GABHAINN, PH.D.,${ }^{c}$ CHRIS ROBERTS, M.SC., ${ }^{d}$ \\ BÉAT WINDLIN, M.A., ${ }^{a}$ ALESSIO VIENO, PH.D., ${ }^{e}$ PERNILLE BENDTSEN, PH.D., $f$ ANNE HUBLET, PH.D., $g$ \\ JORMA TYNJÄLÄ, PH.D., ${ }^{h}$ RAILI VÄLIMAA, PH.D., ${ }^{h}$ ZUZANA DANKULINCOVÁ, PH.D., ${ }^{i}$ KATRIN AASVEE, M.D., PH.D., ${ }^{j}$ \\ ZSOLT DEMETROVICS, PH.D., ${ }^{k}$ JUDIT FARKAS, M.A., ${ }^{k}$ WINFRIED VAN DER SLUIJS, PH.D., ${ }^{l}$ \\ MARGARIDA GASPAR DE MATOS, PH.D., ${ }^{m}$ JOANNA MAZUR, PH.D., ${ }^{n}$ AND MATTHIAS WICKI, M.A. ${ }^{a}$ \\ ${ }^{a}$ Addiction Switzerland, Research Institute, Lausanne, Switzerland \\ ${ }^{b}$ Behavioural Science Institute, Radboud University Nijmegen, the Netherlands \\ ${ }^{c}$ Health Promotion Research Centre, School of Health Sciences, National University of Ireland, Galway, Ireland \\ ${ }^{d}$ Social Research and Information Division, Welsh Government, Cardiff, United Kingdom \\ ${ }^{e}$ Department of Developmental and Social Psychology, University of Padova, Italy \\ ${ }^{f}$ National Institute of Public Health, University of Southern Denmark, Copenhagen, Denmark \\ gDepartment of Public Health, Ghent University, Belgium \\ ${ }^{h}$ Department of Health Sciences, Research Centre for Health Promotion, University of Jyväskylä, Finland \\ ${ }^{i}$ Health Psychology Unit, Institute of Public Health, Medical Faculty, PJ Safarik University, Kosice, Slovak Republic \\ ${ }^{j}$ Chronic Disease Department, National Institute for Health Development, Tallinn, Estonia \\ ${ }^{k}$ Department of Clinical Psychology and Addiction, Institute of Psychology, Eötvös Loránd University, Budapest, Hungary \\ ${ }^{l}$ Child and Adolescent Health Research Unit (CAHRU), School of Medicine, University of St. Andrews, United Kingdom \\ ${ }^{m}$ Department of International Health, Lisbon University, Portugal \\ ${ }^{n}$ Department of Child and Adolescent Health, Institute of Mother and Child, Warsaw, Poland
}

\begin{abstract}
Objective: The purpose of this study was to test the structure and endorsement of drinking motives and their links to alcohol use among 11- to 19-year-olds from 13 European countries. Method: Confirmatory factor analysis, latent growth curves, and multiple regression models were conducted, based on a sample of 33,813 alcohol-using students from Belgium, Denmark, Estonia, Finland, Hungary, Ireland, Italy, Poland, Portugal, Scotland, Slovakia, Switzerland, and Wales who completed the Drinking Motives Questionnaire Revised Short Form (DMQ-R SF). Results: The findings confirmed the hypothesized fourdimensional factor structure. Social motives for drinking were most frequently indicated, followed by enhancement, coping, and conformity motives, in that order, in all age groups in all countries except Finland. This rank order was clearest among older adolescents and those from
\end{abstract}

northern European countries. The results confirmed that, across countries, social motives were strongly positively related to drinking frequency, enhancement motives were strongly positively related to frequency of drunkenness, and conformity motives were negatively related to both alcohol outcomes. Against our expectations, social motives were more closely related to drunkenness than were coping motives, particularly among younger adolescents. Conclusions: The findings reveal striking cross-cultural consistency. Health promotion efforts that are based on, or incorporate, drinking motives are likely to be applicable across Europe. As social motives were particularly closely linked to drunkenness among young adolescents, measures to impede the modeling of alcohol use and skills to resist peer pressure are particularly important in this age group. (J. Stud. Alcohol Drugs, 75, 428-437, 2014)

\begin{abstract}
A DOLESCENCE IS AN IMPORTANT PERIOD in terms of physiological, psychological, and lifestyle changes. It is at this time of vulnerability to the damaging effects of alcohol that drinking onset and the development of specific drinking patterns is initiated. To better understand these behaviors, the concept of motives has been shown to be particularly interesting. Drinking motives are among the

Received: August 28, 2013. Revision: November 19, 2013.

Data collection was funded by each of the participating countries separately. Production of this manuscript was funded mainly by Swiss Foundation for Alcohol Research Grant No. 221 (awarded to Emmanuel Kuntsche).

*Correspondence may be sent to Emmanuel Kuntsche at Addiction Switzerland, Research Institute, P.O. Box 870, 1001 Lausanne, Switzerland, or via email at: EKuntsche@suchtschweiz.ch.
\end{abstract}

most proximal factors for engaging in drinking, the pathways through which other determinants are mediated (Cooper, 1994; Kuntsche et al., 2010b). Cox and Klinger (1988, 1990) argued that individuals drink alcohol either to obtain positive outcomes or to avoid negative ones. They may also be motivated by internal rewards such as the enhancement of a desired emotional state or by external rewards such as social approval. Based on this conceptualization, Cooper (1994) developed the Drinking Motives Questionnaire Revised (DMQ-R) to measure four motive categories combining these two dimensions: enhancement (internal and positive, e.g., drinking to have fun), coping (internal and negative, e.g., drinking to forget problems), social (external and positive, e.g., drinking to be sociable), and conformity (external and negative, e.g., drinking to fit in with a group). 
Until recently, most evidence was drawn from Western industrialized countries such as the United States, Canada, and Switzerland. Only in the past few years have studies in a range of more diverse countries such as Great Britain (Atwell et al., 2011; Bruce et al., 2012), Germany (Wurdak et al., 2010), Hungary (Németh et al., 2011a; Urbán et al., 2008), Italy (Graziano et al., 2010; Mazzardis et al., 2010), the Netherlands (Crutzen and Kuntsche, 2013; SchellemanOffermans et al., 2011), Spain (Mezquita et al., 2011; Németh et al., 2011b), and Sweden (Comasco et al., 2010) investigated the structure of drinking motives and/or their links to alcohol-related outcomes. The evidence from these studies and from previous research is fairly consistent in that adolescents reported that they drank most often for social motives, followed by enhancement, coping, and conformity motives in that order (Cooper, 1994; Kuntsche and Kuntsche, 2009; Kuntsche et al., 2008).

This rank order was found consistently in different age groups across adolescence. However, although enhancement and particularly social motives were only slightly more likely to be reported than coping and conformity motives in early and mid-adolescence, the differences were more prominent in older age groups (Cooper, 1994; Kuntsche et al., 2006). It appears that motivation to engage in drinking becomes more distinct across the adolescent years (Schelleman-Offermans et al., 2011).

With respect to links to alcohol use, studies have shown that social motives are positively related to frequent but moderate drinking, whereas internal motives (i.e., enhancement and coping) are more closely related to higher levels of risky drinking. Unlike all other motive dimensions, conformity motives have been found to be inversely related to both types of alcohol use when the other three motive dimensions were taken into account (Cooper, 1994; Cooper et al., in press; Kuntsche et al., 2005).

The degree to which the structure of drinking motives and links to alcohol are similar or differ across Europe is not yet well understood. To our knowledge, only one study has compared the structure and endorsement of drinking motives and their links to alcohol outcomes between two European countries (i.e., Hungary and Spain; Németh et al., 2011b). Consistent with another cross-cultural drinking motives study that compared adolescents from a European country (Switzerland) with those living in North America (Kuntsche et al., 2008), Németh and colleagues (2011b) confirmed the four-dimensional motive structure and the rank order (social $>$ enhancement $>$ coping $>$ conformity) in both countries. However, this rank order was stronger in Hungary. That is, Hungarian participants scored higher on social, enhancement, and coping motives than those in Spain, with no difference for conformity motives.

Among the possible explanations for these differences is the traditional drinking culture in northern Europe, where excessive drinking on occasions, particularly on weekends, is more socially acceptable (e.g., considered a "leisure" behavior). This differs considerably from that in the southern European, mostly wine-producing countries, where the predominant drinking pattern is frequent consumption of moderate amounts of alcohol, often accompanying meals (Kuntsche et al., 2004; Room, 2001).

Despite these advances in our understanding, differences in data collection methods, sample characteristics (including age range), analytic strategies, and outcome variables mean that it is difficult to compare results across studies. Based on a sample from 13 countries across Europe comprising 33,813 alcohol-using adolescents that together cover the age range from early to late adolescence (ages 11-19 years), the present study aimed to test the following hypotheses: (a) the four-dimensional factor structure can be confirmed by using the DMQ-R Short Form (DMQ-R SF) (Kuntsche and Kuntsche, 2009); (b) social motives are most frequently indicated followed by enhancement, coping, and conformity motives; (c) social motives are associated with drinking frequency but not with drunkenness frequency; (d) enhancement motives, followed by coping motives, are most strongly positively related to the frequency of drunkenness; (e) conformity motives are inversely related to both drinking and drunkenness frequency; and (f) hypotheses (a) through (e) hold in all adolescent age groups across all 13 countries.

\section{Method}

\section{Study design}

Most data for this study were collected through the Health Behaviour in School-aged Children (HBSC) study, a World Health Organization (Europe) collaborative project. Of the 43 countries participating in the 2009/2010 survey, 11 agreed to include the DMQ-R SF. The Hungarian and Italian data were collected independently from HBSC but using a similar procedure. Data were collected between autumn 2008 and spring 2010. In each country, nationally representative surveys were conducted, with the exception of Belgium (data representative for the Flemish Community), Hungary (data representative for the Budapest region), and Italy (data taken from one part of the Veneto region).

For all samples, students were selected using a clustered sampling design, where either classes or schools served as primary sampling units. Data were collected by anonymous self-report questionnaires distributed in the classroom. Each study was approved by the appropriate ethics review board. The overall response rate (including dropouts and nonresponse at the individual, class, and school levels) was $60 \%$ or higher for all HBSC countries, except Denmark (46\%) and Flemish Belgium (29\%). For the Hungarian sample, it was $76 \%$, and for the Italian sample, $93 \%$. 


\section{Sample}

The gross sample for this study consisted of 62,138 students between 11 and 19 years old. Cases with missing values for gender or age (about 1\% in total, ranging from $0 \%$ in Portugal to $6 \%$ in Denmark) were excluded at the country level. Because only students who had consumed alcohol can answer questions on drinking motives, the 27,797 (44.7\%) students who had not consumed alcohol in the last 12 months were excluded from the sample. Another 528 $(0.8 \%)$ cases were excluded because they had missing values in all three items of at least one of the four different motive dimensions. The remaining net sample used in the analyses consisted of 33,813 students.

\section{Measures}

Drinking motives. The 12-item DMQ-R SF (Kuntsche and Kuntsche, 2009) was used to assess enhancement, social, conformity, and coping motives for drinking in the last 12 months. Each dimension comprises three items and was rated on a relative frequency scale. In Belgium, Finland, Hungary, Portugal, Scotland, Slovakia, and Switzerland, the five-point scale from the original DMQ-R (Cooper, 1994) was used: (almost) never (1), some of the time (2), about half of the time (3), most of the time (4), and (almost) always (5). In Denmark, Estonia, Ireland, Italy, Poland, and Wales, the original three-point scale of the DMQ-R SF was used. Those values were transformed to match those of the DMQ-R as follows: (almost) never (coded as 1), about half of the time (coded as 3), and (almost) always (coded as 5) (see Kuntsche et al., 2008, for a similar procedure). Comparing neighboring countries showed that combining the three- and five-point scales is unlikely to have an impact on the results presented here.

Sociodemographic variables. Gender was coded 0 for girls and 1 for boys. Year and month of birth was used to calculate participants' age. To investigate differences between non-wine-producing countries in northern Europe and wine-producing countries in southern and central Europe (Room, 2001), half of the countries were classified as northern European countries (Denmark, Estonia, Finland, Ireland, Poland, Scotland, and Wales; coded as 1) and half as nonnorthern countries mostly from southern and central Europe (Belgium, Hungary, Italy, Portugal, Slovakia, and Switzerland; coded as 0 ).

Drinking frequency. To measure the frequency of alcohol use, participants were asked how often they drank beer, wine, alcopops, spirits, up to two other alcoholic drinks (depending on the choice of the participating countries), as well as any other drink that contains alcohol. Answer categories ranged from never to every day for each of these items and were recoded to an annual frequency (e.g., every week was coded as 52). To determine drinking frequency, the most commonly reported alcoholic beverage was used. As an exception, in the Hungarian survey, only data on drinking in the past 30 days were available. Because no comparable question was asked in the Polish survey, Polish data were excluded from the drinking frequency analyses.

Drunkenness frequency. Participants were asked, "Have you ever had so much alcohol that you were really drunk?" with answer categories no, never; yes, once; yes, 2-3 times; yes, 4-10 times; and yes, more than 10 times. To create a linear frequency measure, midpoints of categories were used and 13 was adopted for the upper category (10 times plus the range to midpoint of adjacent category). In the Hungarian survey, participants were asked whether they had ever been drunk, and the answers were coded dichotomously. Because no comparable question was asked in the Italian survey, Italian data were excluded from the drunkenness frequency analyses.

\section{Analytic strategy}

Because the entire age range was not assessed in each of the countries (Table 1), the analyses were conducted separately in the three age groups: 11- to 13-year-olds, 14- to 16-year-olds, and 17- to 19-year-olds. These groups represent early, middle, and late adolescence (Rice and Dolgin, 2002), and analyzing these groups separately enables a more thorough comparison of findings across countries. All analyses were conducted using Mplus 7 statistical software (Muthén and Muthén, 1998-2012) and adjusted for the clustered design effect associated with sampling school classes.

To confirm the four-factor structure of drinking motives, confirmatory factor analysis was used. For each country and age group individually, the comparative fit index (CFI) and standardized root mean square residual (SRMR) were used as indices of model fit. CFI values close to 1 (higher than .90 , preferably close to .95 ) and SRMR values close to 0 (lower than .10, preferably close to .08) indicate good model fit (Hooper et al., 2008; Kline, 2005; Marsh et al., 2004). In cases of poor model fit, alternative three- and two-factor models were tested. Internal consistency for each drinking motive was assessed using Cronbach's $\alpha$, where values greater than or equal to .70 were considered as acceptable (Nunnally and Bernstein, 1994).

Latent growth curve modeling (LGCM) was used to test the order of motive endorsement within individuals. Social motives were hypothesized to be the most frequently indicated motive dimension and were set as the intercept, with a slope indicating the assumed decrease from social to enhancement to coping to conformity motives being estimated. To test inter-individual differences in the level and order of the four motive dimensions, the intercept and the slope were regressed on age, gender, and region.

To investigate associations with alcohol use, multiple regression models were estimated with the drinking motive 
KUNTSCHE ET AL.

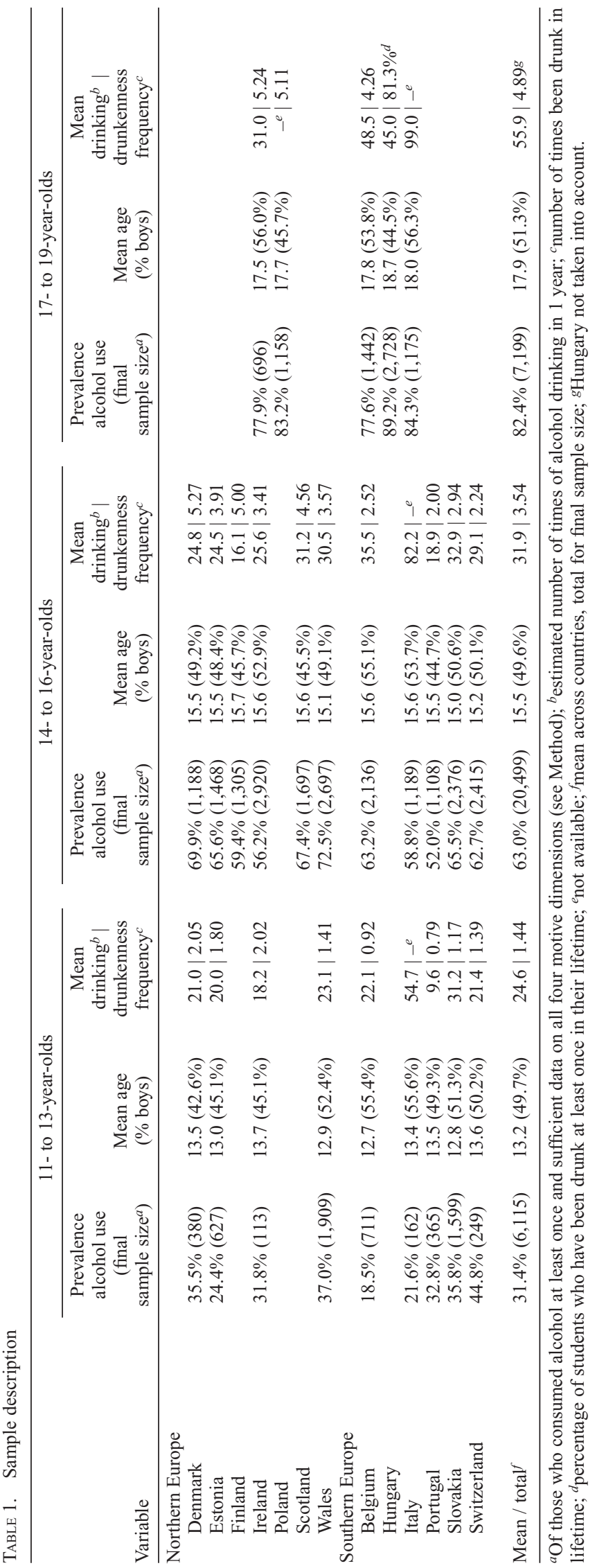


TABLE 2. Model fit indices of the confirmatory factor analysis and internal consistencies (Cronbach's $\alpha$ values) of the four drinking motive dimensions according to age group

\begin{tabular}{|c|c|c|c|c|c|c|}
\hline \multirow[b]{2}{*}{ Variable } & \multicolumn{2}{|c|}{11 - to 13 -year-olds } & \multicolumn{2}{|c|}{ 14- to 16 -year-olds } & \multicolumn{2}{|c|}{17 - to 19 -year-olds } \\
\hline & CFI & SRMR & CFI & SRMR & CFI & SRMR \\
\hline Across countries & .96 & .034 & .96 & .037 & .95 & .034 \\
\hline \multicolumn{7}{|l|}{ Northern Europe } \\
\hline Denmark & .93 & .043 & .98 & .033 & & \\
\hline Estonia & .94 & .043 & .91 & .064 & & \\
\hline Finland & & & .96 & .039 & & \\
\hline Ireland & .92 & .064 & .96 & .037 & .96 & .045 \\
\hline Poland & & & & & .95 & .042 \\
\hline Scotland & & & .97 & .034 & & \\
\hline Wales & .96 & .036 & .96 & .038 & & \\
\hline \multicolumn{7}{|l|}{ Southern Europe } \\
\hline Belgium & .94 & .047 & .93 & .058 & .95 & .053 \\
\hline Hungary & & & & & .96 & .038 \\
\hline Italy & .92 & .077 & .94 & .046 & .96 & .044 \\
\hline Portugal & .93 & .050 & .90 & .075 & & \\
\hline Slovakia & .96 & .033 & .95 & .037 & & \\
\hline Switzerland & .96 & .061 & .96 & .036 & & \\
\hline \multicolumn{7}{|c|}{ Internal consistencies ${ }^{a}$} \\
\hline Social & .77 & & .82 & & .80 & \\
\hline Enhancement & .72 & & .74 & & .64 & \\
\hline Coping & .82 & & .84 & & .81 & \\
\hline Conformity & .81 & & .80 & & .75 & \\
\hline
\end{tabular}

Notes: $\mathrm{CFI}=$ comparative fit index; $\mathrm{SRMR}=$ standardized root mean residual. ${ }^{a}$ Across countries (country-specific results are available from the authors upon request).

dimensions as separate independent variables and frequency of drinking and drunkenness as dependent variables. To test differences in these links across age groups (11-13, 14-16, and 17-19 years old), between boys and girls and between southern and northern Europe, interaction terms with the four motive dimensions were included in the models. To approximate a normal distribution and to reduce the impact of extreme values, both alcohol use variables were logtransformed (Tabachnick and Fidell, 2001), except for the dichotomous drunkenness indicator in Hungary, for which a logistic regression function was used.

\section{Results}

\section{Sample description}

Among the 11- to 13-year-olds, almost one third had consumed alcohol and were included in the study (Table 1). Almost two thirds and more than four fifths of 14- to 16-year-olds and 17- to 19-year-olds, respectively, were included based on their alcohol consumption. On average, those in the youngest group reported drinking 25 times in the last year and reported being drunk 1.5 times. There was considerable variation across the countries, with, on average, less than 10 drinking occasions in Portugal and more than 50 drinking occasions in Italy (both southern European countries). In Belgium and Portugal, participants in the 11to 13-year-old group reported being drunk on average less than once, whereas in Denmark and Ireland they reported on average two occasions of drunkenness. Among those ages 14-16 years, those in northern Europe (in Denmark, Finland, and Scotland) had a higher frequency of drunkenness than their southern European counterparts (in Portugal and Switzerland). In contrast, fewer cross-national differences were found in reported drinking frequency.

\section{Confirming the four-factor structure}

Taking all countries together, the CFI values were above the .95 threshold and the SRMR values were below the .05 threshold in all age groups (Table 2). Similarly, when looking at the different age groups across the different countries, both the CFI and the SRMR indicated a good model fit. Only two exceptions were found, among 14- to 16-year-olds in Estonia and Portugal. However, even in these cases, the four-factor model had a much better fit (Table 2) than the three-factor model (social and enhancement combined: Estonia: $\mathrm{CFI}=.90, \mathrm{SRMR}=.067$; Portugal: $\mathrm{CFI}=.89, \mathrm{SRMR}$ $=.083$ ) or any of the tested two-factor models (positive vs. negative: Estonia: $\mathrm{CFI}=.85, \mathrm{SRMR}=.066$; Portugal: $\mathrm{CFI}=$ $.70, \mathrm{SRMR}=.126$; internal vs. external: Estonia: $\mathrm{CFI}=.81$, $\mathrm{SRMR}=.072$; Portugal: $\mathrm{CFI}=.70, \mathrm{SRMR}=.156$ ).

\section{Frequency of motive endorsement}

Table 3 shows that, in all countries and age groups, social motives were the most frequently indicated motive dimension, followed by enhancement, coping, and conformity mo- 
TABLE 3. Means of the four drinking motive dimensions according to age group and country

\begin{tabular}{|c|c|c|c|c|c|c|c|c|c|c|c|c|c|c|c|}
\hline \multirow[b]{2}{*}{ Country } & \multicolumn{5}{|c|}{ 11- to 13 -year-olds } & \multicolumn{5}{|c|}{ 14- to 16 -year-olds } & \multicolumn{5}{|c|}{17 - to 19 -year-olds } \\
\hline & Soc. & Enh. & Cop. & Cnf. & Slope & Soc. & Enh. & Cop. & Cnf. & Slope & Soc. & Enh. & Cop. & Cnf. & Slope \\
\hline \multicolumn{16}{|l|}{ Northern } \\
\hline \multicolumn{16}{|l|}{ Europe } \\
\hline Denmark & 2.53 & 2.43 & 1.44 & 1.36 & $-.42 * * *$ & 3.42 & 3.34 & 1.54 & 1.40 & $-.71 * * *$ & & & & & \\
\hline Estonia & 2.58 & 2.50 & 2.31 & 1.98 & $-.19 * * *$ & 3.13 & 3.11 & 2.56 & 1.99 & $-.38 * * *$ & & & & & \\
\hline Finland & & & & & & 3.07 & 3.23 & 1.97 & 1.66 & $-.47 * * *$ & & & & & \\
\hline Ireland & 2.65 & 2.22 & 1.77 & 1.70 & $-.11 * *$ & 3.30 & 2.62 & 1.85 & 1.60 & $-.58 * * *$ & 3.68 & 2.85 & 1.88 & 1.55 & $-.73 * * *$ \\
\hline Poland & & & & & & & & & & & 3.12 & 2.11 & 2.11 & 1.35 & $-.56^{* * *}$ \\
\hline Scotland & & & & & & 3.27 & 2.48 & 1.82 & 1.46 & $-.60 * * *$ & & & & & \\
\hline Wales & 2.58 & 1.90 & 1.79 & 1.60 & $-.30 * * *$ & 3.27 & 2.45 & 2.12 & 1.54 & $-.57 * * *$ & & & & & \\
\hline \multicolumn{16}{|l|}{ Southern } \\
\hline \multicolumn{16}{|l|}{ Europe } \\
\hline Belgium & 1.92 & 1.78 & 1.37 & 1.33 & $-.21 * * *$ & 2.57 & 2.15 & 1.47 & 1.38 & $-.41 * * *$ & 2.67 & 2.24 & 1.51 & 1.32 & $-.47 * * *$ \\
\hline Italy & 2.26 & 1.87 & 1.59 & 1.39 & $-.28 * * *$ & 2.69 & 2.23 & 1.85 & 1.34 & $-.45^{* * *}$ & 2.84 & 2.44 & 2.00 & 1.28 & $-.52 * * *$ \\
\hline Portugal & 2.67 & 2.58 & 2.24 & 2.05 & $-.22 * * *$ & 2.55 & 2.39 & 1.87 & 1.50 & $-.37 * * *$ & & & & & \\
\hline Hungary & & & & & & & & & & & 2.72 & 1.91 & 1.66 & 1.25 & $-.44 * * *$ \\
\hline Slovakia & 2.01 & 1.87 & 1.79 & 1.59 & $-.14 * * *$ & 2.41 & 2.28 & 1.97 & 1.49 & $-.30 * * *$ & & & & & \\
\hline Switzerland & 2.31 & 1.99 & 1.43 & 1.19 & $-.38 * * *$ & 2.60 & 2.31 & 1.60 & 1.18 & $-.48 * * *$ & & & & & \\
\hline
\end{tabular}

Notes: Standard errors not shown but available from the main author upon request. Soc. $=$ social, enh. $=$ enhancement, $c o p .=$ coping, $c$ fnf. $=$ conformity. ${ }^{* *} p<.01 ; * * p<.001$.

tives, in that order. The significant negative slopes emerging from the latent growth curve analysis confirm this trend in motive endorsement. The only exception was 14 - to 16 -yearolds in Finland, who scored on average slightly higher on enhancement than on social motives. However, the general trend was the same in that the overall slope was also significantly negative.

LGCM analysis revealed that there was not only a higher motive endorsement in the older age groups $\left(B_{\text {intercept }}=.194\right.$, $S E=.009, p<.001)$ and in northern European countries $\left(B_{\text {in }}\right.$ tercept $=.313, S E=.009, p<.001)$ but also that the rank order was more pronounced (indicating a larger difference between social/enhancement motives and conformity motives) in older age groups $\left(B_{\text {slope }}=-.294, S E=.010, p<.001\right)$ and in northern European countries $\left(B_{\text {slope }}=-.253, S E=.011, p<\right.$ .001) than in the younger age groups and the southern European countries, respectively. The endorsement of motives was stronger $\left(B_{\text {intercept }}=.065, S E=.009, p<.001\right)$ among boys than girls, but there were no significant gender differences in the rank order $\left(B_{\text {slope }}=-.014, S E=.009, p<.001\right)$.

\section{Links to alcohol use}

When all countries and age groups were combined, social, enhancement, and coping motives were significantly positively related, and conformity motives were significantly negatively related to both measures of alcohol use (Table 4). For drinking frequency, social motives showed the strongest link, whereas for drunkenness it was enhancement motives.

Drinking frequency and drunkenness frequency increased across the age groups. The link between the four motive dimensions and alcohol use was stable across all age groups for drinking frequency but not for drunkenness frequency. The strong effect of social (main effect: $\beta=.42$ ) and conformity motives (main effect: $\beta=-.21$ ) found in the youngest age group was weaker in the older age groups (interaction social: $\beta=-.24$, interaction conformity: $\beta=.07$ ), whereas the effect of enhancement motives (main effect: $\beta=.15$ ) was stronger in the older age groups (interaction: $\beta=.23$ ).

Even when drinking motives were taken into account, boys drank significantly more frequently and were drunk more often than girls (except among 14- to 16-year-olds). In the youngest group (11- to 13-year-olds), there were no significant gender differences in the link between the four motive dimensions and drinking frequency. In the two older age groups, the link between enhancement motives and drinking frequency was stronger for boys than for girls. In the two younger age groups, the link between coping motives and drunkenness frequency was weaker for boys than for girls. Among 14- to 16-year-olds, the strong link between enhancement motives and drunkenness frequency was even stronger for boys than for girls.

Drinking frequency in all age groups and drunkenness frequency in the youngest age group (11- to 13-year-olds) were significantly lower among students in northern Europe than among those in southern Europe. However, the strong negative link between conformity motives and drinking frequency found for the youngest age group in southern European countries was significantly weaker in northern European countries. In the two older groups, the strong positive link between enhancement motives and drinking frequency found in southern European countries was significantly weaker in northern European countries, but the effect of social motives was stronger in the northern than in the southern countries among the 17- to 19 -year-olds. 
TABLE 4. Alcohol use regressed on age, gender, region, and drinking motives

\begin{tabular}{|c|c|c|c|c|c|c|c|c|}
\hline \multirow[b]{2}{*}{ Variable } & \multirow[b]{2}{*}{ Overall } & \multirow[b]{2}{*}{ Age } & \multicolumn{2}{|c|}{ 11- to 13 -year-olds } & \multicolumn{2}{|c|}{ 14- to 16 -year-olds } & \multicolumn{2}{|c|}{ 17- to 19 -year-olds } \\
\hline & & & Gender $^{a}$ & Location $^{b}$ & Gender $^{a}$ & Location $^{b}$ & Gender $^{a}$ & Location $^{b}$ \\
\hline \multicolumn{9}{|c|}{ Drinking frequency } \\
\hline Main effect & & $.25 * * *$ & $.08 *$ & $-.16 * * *$ & .04 & $-.11 * * *$ & $.11 *$ & $-.31 * * *$ \\
\hline Soc. & $.21 * * *$ & $.12 * * *$ & $.18^{* * *}$ & $20 * * *$ & $.18^{* * *}$ & $.23 * * *$ & $.10 * * *$ & $.13 * * *$ \\
\hline Enh. & $.13 * * *$ & $.09 *$ & $.16^{* * *}$ & $.15 * * *$ & $.12 * * *$ & $.22 * * *$ & $.14 * * *$ & $.25 * * *$ \\
\hline Cop. & $.14 * * *$ & $.12 * * *$ & $.10 * * *$ & $.10 * *$ & $.18^{* * *}$ & $.13 * * *$ & $.18 * * *$ & $.15 * * *$ \\
\hline Cnf. & $-.12 * * *$ & -.03 & $-.14 * * *$ & $-.23 * * *$ & $-.11 * * *$ & $-.09 * * *$ & $-.11 * * *$ & $-.08 * * *$ \\
\hline Int. $\times$ Soc. & & .03 & -.00 & -.02 & .00 & .02 & -.07 & $.13 *$ \\
\hline Int. $\times$ Enh. & & .05 & -.00 & -.00 & $.09 * * *$ & $-.12 * * *$ & $.11 *$ & $-.10 *$ \\
\hline Int. $\times$ Cop. & & .03 & -.00 & .00 & -.02 & .03 & .01 & -.05 \\
\hline Int. $\times$ Cnf. & & -.06 & -.01 & $.18^{* * *}$ & .03 & .04 & .02 & .05 \\
\hline$R^{2}$ & $12.5 \%$ & $19.6 \%$ & $10.1 \%$ & $10.4 \%$ & $15.0 \%$ & $16.5 \%$ & $14.5 \%$ & $18.8 \%$ \\
\hline \multicolumn{9}{|l|}{$\begin{array}{l}\text { Drunkenness } \\
\text { frequency }\end{array}$} \\
\hline Main effect & & $.11^{* * *}$ & $.07 *$ & $-.16^{* * *}$ & $.05^{* *}$ & .00 & $.09 *$ & -.05 \\
\hline Soc. & $.27 * * *$ & $.42 * * *$ & $29 * * *$ & $.34 * * *$ & $.25^{* * *}$ & $.22 * * *$ & $.17 * * *$ & $.04 *$ \\
\hline Enh. & $.34 * * *$ & $.15 * * *$ & $.25 * * *$ & $.15 * * *$ & $.34 * * *$ & $.34 * * *$ & $.28 * * *$ & $.38 * * *$ \\
\hline Cop. & $.12 * * *$ & $.14 * * *$ & $.19 * * *$ & $.12 * * *$ & $.17 * * *$ & $.15 * * *$ & $.13 * * *$ & $-.04 *$ \\
\hline Cnf. & $-.16^{* * *}$ & $-.21 * * *$ & $-.21 * * *$ & $-.23 * * *$ & $-16^{* * * *}$ & $-.16 * * *$ & $-.13 * * *$ & $-.07 * * *$ \\
\hline Int. $\times$ Soc. & & $-.24 * * *$ & .06 & $-.10 *$ & -.01 & .04 & -.01 & $.35^{* * *}$ \\
\hline Int. $\times$ Enh. & & $.23 * * *$ & -.04 & $.19 * * *$ & $.05 *$ & .04 & .08 & $-.10^{*}$ \\
\hline Int. $\times$ Cop. & & -.02 & $-.12 * *$ & .03 & $-.07 * * *$ & -.03 & -.06 & $.22 * * *$ \\
\hline Int. $\times$ Cnf. & & $.07 * *$ & $.08^{*}$ & $.13 * *$ & .03 & .02 & .02 & $-.09^{*}$ \\
\hline$R^{2}$ & $32.7 \%$ & $34.2 \%$ & $25.7 \%$ & $26.4 \%$ & $34.2 \%$ & $34.2 \%$ & $23.8 \%$ & $30.6 \%$ \\
\hline
\end{tabular}

Notes: Shown are standardized regression coefficients (betas). ${ }^{a}$ Girls $=0$, boys $=1 ;{ }^{b}$ southern $/$ central Europe $=0$, northern Europe $=1$. Soc. $=$ social; enh. $=$ enhancement; cop. $=$ coping; cnf. $=$ conformity; Int. $\times=$ interaction with; $R^{2}=$ explained variance .

$* p<.05 ; * * p<.01 ; * * * p<.001$.

For drunkenness frequency among 11 - to 13 -year-olds, the effect was stronger for enhancement and conformity motives and weaker for social motives among students in northern Europe than those from southern Europe. No regional differences between the four motive dimensions and frequency of drunkenness were found in the middle age group (14- to 16-year-olds). In the oldest group (17- to 19-year-olds), there was a stronger effect on drunkenness for social and coping motives and a weaker effect for enhancement motives in the northern than in the southern European countries.

\section{Discussion}

The aim of this study was to test for similarities and differences in the factor structure and rank order of drinking motives and their links to alcohol use among 11- to 19-yearolds from 13 European countries.

\section{Factor structure}

With an overall CFI of at least .95 and an SRMR of .034 and consistent with previous research (Kuntsche and Kuntsche, 2009; Mazzardis et al., 2010; Németh et al., 2011a, 2011b), the results showed a good model fit of the DMQ-R SF across age groups. It was only in some countries (e.g., Estonia and Portugal) that the model fit was somewhat poorer. It is possible that language connotations could be responsible in part for a lower model fit. That being said, the four-factor solution remained the best-fitting factor structure in all countries.

Cronbach's $\alpha$ values above .70 demonstrated good internal consistency, particularly so when considering that only three items were used to measure each motive dimension (Nunnally and Bernstein, 1994). The only exception was enhancement motives in the oldest age group, indicating that when entering adulthood, drinking for enhancement becomes less homogeneous (Crutzen and Kuntsche, 2013; Mazzardis et al., 2010; Németh et al., 2011a). To increase positive internal emotional states, some people might continue drinking to seek extreme sensations such as drunkenness, whereas others might drink because they enjoy the taste.

\section{Motive endorsement}

The fact that drinking motives are closely related to personality traits (Cooper et al., in press; Kuntsche et al., 2006) that have been shown to be very consistent across countries, cultures, languages, age groups, and life stages (Allik, 2005; Heine and Buchtel, 2009; Roberts and DelVecchio, 2000) is one explanation for the highly consistent rank order of social motives followed by enhancement, coping, and conformity. Of the 75 comparisons summarized in Table 3, the 
documented rank order was violated only once. Among 14- to 16-year-old Finns, the average level of enhancement motives was slightly higher than that for social motives. One explanation might be the traditional Scandinavian drinking culture, where drinking to have fun and to get drunk is more socially acceptable (Kuntsche et al., 2004; Room, 2001).

A more pronounced rank order was found in older age groups. Moving from early to late adolescence, young people spend more and more time with peers, and risk taking and sensation seeking become widespread (Steinberg, 2008). This might explain why social and enhancement motives, in particular, were more frequently indicated across the age groups.

A similar pattern emerged for geographical region, where the levels of social and enhancement motives were higher in northern than in southern European countries (expressed through a significantly higher intercept and a steeper negative slope). The higher frequency of peer contacts (particularly in the evening, when adult supervision is less likely; Currie et al., 2012), fewer demands and rules from parents (Claes et al., 2011), and the more permissive drinking culture (Kuntsche et al., 2004; Room, 2001) may help to explain these findings.

\section{Links to alcohol use}

The results confirmed previous findings (Cooper et al., in press; Kuntsche et al., 2005; Kuntsche and Kuntsche, 2009; Németh et al., 2011a) that, across countries, social motives were strongly positively related to drinking frequency, enhancement motives were strongly positively related to frequency of drunkenness, and conformity motives were negatively related to both alcohol outcomes. The finding that social motives were more closely related to drunkenness than were coping motives was not anticipated. This pattern was particularly notable among the younger age groups. Previous research has often been conducted with older adolescents or young adults. It appears that, particularly for young adolescents, drunkenness occurs most frequently among those who are more likely to drink at social gatherings, parties, and celebrations (Kuntsche and Müller, 2012).

In older age groups, the strength of the association between drunkenness and social motives was lower, whereas for enhancement motives it was higher (see interaction in Table 4). This is consistent with longitudinal research showing that only enhancement motives predicted young adults' excessive alcohol consumption on weekends (Kuntsche and Cooper, 2010). It appears that younger adolescents are more responsive to their environment and sensitive to external social rewards than older adolescents, who are more likely to drink to change internal emotional states (SchellemanOffermans et al., 2011).

Despite the very robust finding that males drink more frequently and more excessively than females (Currie et al., 2012; Kuntsche et al., 2004), only one gender difference was identified. The link between enhancement motives and drinking frequency was significantly stronger among 14- to 16- and 17- to 19-year-old boys than girls, whereas the link between coping motives and drunkenness frequency was significantly stronger among 11- to 13- and 14- to 16-yearold girls. This is consistent with research (Kuntsche et al., 2006) showing that males are more likely to be extroverted and impulsive and to seek extreme sensations (e.g., through enhancement drinking), whereas females are more avoidant and anxiety-fearing, which may predispose them to drink alcohol to cope with these unpleasant emotions.

Concerning regional differences, it appears that effects of enhancement and conformity motives are stronger and those of social motives are weaker among students ages 11-13 years in northern Europe than in southern Europe, whereas the opposite is the case for the 17- to 19-year-olds. However, fewer countries provided data for the oldest age group. Consequently, these results might reflect country-specific issues rather than broad geographical differences in drinking culture. Interestingly, although there was a higher frequency of drinking and drunkenness in northern countries, the regional main effect in the multiple regression model was negative, indicating that at least some of the regional differences in alcohol use were mediated by differences in motives. Further research will be required to investigate this in more detail.

\section{Limitations, strengths, and future research directions}

Among the limitations is the cross-sectional nature of the data that obviates causal conclusions. All data were based on self-reports that are subject to recall bias and only roughly correspond to behavioral measures (Comasco et al., 2009; Gmel and Rehm, 2004). However, collecting breath alcohol analysis data, for example, in an international study across thousands of adolescents would inevitably be difficult to administer and very costly. Although most of the countries included used a standardized research protocol (i.e., the same questionnaire, sampling procedure, and so on) and deployed a translation-back translation process, some results may be affected by methodological differences. Some countries (e.g., Hungary and Italy) were only represented by local samples, some countries had a low response rate at the school level (e.g., Flemish Belgium and Denmark), and not all age groups were sampled in all countries. To our knowledge, however, both in terms of sample size and the variety of countries included, this is the largest study in more than 50 years of drinking motive research enabling the testing of gender, age, and cultural differences. Because this study focused only on Europe, it would be interesting to extend this large-scale cross-cultural comparison to countries from other continents. Comparisons of drinking motives between the United States and a country from Africa (Gire, 2002) and from Asia (Perera and Torabi, 2012) are promising examples in this respect. 


\section{Conclusion}

Having confirmed the four-dimensional factor structure and the rank order of motive endorsement in all age groups across countries, the findings presented here reveal striking cross-cultural similarities across the 13 European countries included in this study. The DMQ-R and its short form were found to be useful and valid instruments for different purposes such as research, screening, and intervention (e.g., Conrod et al., 2006; Kuntsche et al., 2010a; Stewart et al., 2005) and in different countries (e.g., Mazzardis et al., 2010; Németh et al., 2011a; Schelleman-Offermans et al., 2011; Wurdak et al., 2010). This study provided further evidence that the instruments can be used to compare various countries cross-culturally (Kuntsche et al., 2008). For prevention, this means that health promotion efforts that are based on or incorporate drinking motives are likely to be applicable throughout Europe, North America, and possibly other countries.

Despite the fact that the factor structure and rank order of drinking motives was confirmed among both younger and older adolescents, some age differences were found that have important implications for successful preventive action. Whereas social motives have been found to be related to moderate drinking in late adolescence and in adulthood (Gmel et al., 2012; Kuntsche et al., 2005), we found a particularly strong relationship between social motives and drunkenness in the youngest age group. Younger adolescents appear to be particularly responsive to drinking in their environment and sensitive to external social rewards (Schelleman-Offermans et al., 2011; Steinberg, 2008), and their excessive drinking is often motivated by drinking in social situations (parties, celebrations, etc.; Kuntsche and Müller, 2012). Intervention approaches focusing on social influences, such as normative education or resistance skills training to impede the modeling of alcohol use and to reinforce resistance to offers of alcohol by peers (Botvin, 2000), are likely to be particularly effective in this age group. In addition, for parents in northern European countries, it seems important to set, communicate, and reinforce restrictive rules concerning underage drinking and to promote supervised adult-guided leisure time activities (Koutakis et al., 2008). Moreover, discussion of drinking motives might also be useful in preclinical settings across Europe, such as by social assistants and psychologists, to better understand the reasons promoting and maintaining alcohol consumption, as well as for developing future prevention and intervention strategies.

\section{Acknowledgments}

HBSC is an international study carried out in collaboration with the World Health Organization (Europe). The international coordinator of the 2009/2010 study was Professor Candace Currie, University of St. Andrews, Scotland, and the data bank manager was Professor Oddrun Samdal, University of Bergen, Norway.
Data from the following countries were included in the present study (principal investigators in parentheses): Flemish-speaking Belgium (Carine Vereecken), Denmark (Pernille Due), Estonia (Katrin Aasvee), Finland (Jorma Tynjälä), Ireland (Saoirse Nic Gabhainn), Poland (Joanna Mazur), Portugal (Margarida Gaspar De Matos), Slovakia (Andrea Madarasova Geckova), Switzerland (Emmanuel Kuntsche), Scotland (Candace Currie), and Wales (Chris Roberts). Zsolt Demetrovics and Massimo Santinello were responsible for the non-HBSC surveys in Hungary and Italy, respectively.

All authors disclose that they do not have any conflicts of interest, including specific financial interests and relationships or affiliations (other than those affiliations listed in the title page of the manuscript) relevant to the subject of this article. The corresponding author had full access to all the data in the study and takes responsibility for the integrity of the data and the accuracy of the data analysis.

\section{References}

Allik, J. (2005). Personality dimensions across cultures. Journal of Personality Disorders, 19, 212-232.

Atwell, K., Abraham, C., \& Duka, T. (2011). A parsimonious, integrative model of key psychological correlates of UK university students' alcohol consumption. Alcohol and Alcoholism, 46, 253-260.

Botvin, G. J. (2000). Preventing drug abuse in schools: Social and competence enhancement approaches targeting individual-level etiologic factors. Addictive Behaviors, 25, 887-897.

Bruce, G., Curren, C., \& Williams, L. (2012). Alexithymia and alcohol consumption: The mediating effects of drinking motives. Addictive Behaviors, 37, 350-352.

Claes, M., Perchec, C., Miranda, D., Benoit, A., Bariaud, F., Lanz, M., . . . Lacourse, É. (2011). Adolescents' perceptions of parental practices: A cross-national comparison of Canada, France, and Italy. Journal of Adolescence, 34, 225-238.

Comasco, E., Berglund, K., Oreland, L., \& Nilsson, K. W. (2010). Why do adolescents drink? Motivational patterns related to alcohol consumption and alcohol-related problems. Substance Use \& Misuse, 45, 1589-1604.

Comasco, E., Nordquist, N., Leppert, J., Oreland, L., Kronstrand, R., Alling, C., \& Nilsson, K. W. (2009). Adolescent alcohol consumption: Biomarkers PEth and FAEE in relation to interview and questionnaire data. Journal of Studies on Alcohol and Drugs, 70, 797-804.

Conrod, P. J., Stewart, S. H., Comeau, N., \& Maclean, A. M. (2006). Efficacy of cognitive-behavioral interventions targeting personality risk factors for youth alcohol misuse. Journal of Clinical Child and Adolescent Psychology, 35, 550-563.

Cooper, M. L. (1994). Motivations for alcohol use among adolescents: Development and validation of a four-factor-model. Psychological Assessment, 6, 117-128.

Cooper, M. L., Kuntsche, E., Levitt, A., Barber, L., \& Wolf, S. (in press). A motivational perspective on substance use: Review of theory and research. In K. J. Sher (Ed.), Oxford handbook of substance use disorders. New York, NY: Oxford University Press.

Cox, W. M., \& Klinger, E. (1988). A motivational model of alcohol use. Journal of Abnormal Psychology, 97, 168-180.

Cox, W. M., \& Klinger, E. (1990). Incentive motivation, affective change, and alcohol use: A model. In W. M. Cox (Ed.), Why people drinkParameters of alcohol as a reinforcer (pp. 291-314). New York, NY: Gardner Press.

Crutzen, R., \& Kuntsche, E. (2013). Validation of the four-dimensional structure of drinking motives among adults. European Addiction Research, 19, 222-226.

Currie, C., Zanotti, C., Morgan, A., Currie, D., De Looze, M., Roberts, C., . . . Barnekow, V. (2012). Social determinants of health and wellbeing among young people. Health Behaviour in School-aged Children (HBSC) study: International report from the 2009/2010 survey. Retrieved from http:/www.euro.who.int/en/what-we-publish/abstracts/ 
social-determinants-of-health-and-well-being-among-young-people.health-behaviour-in-school-aged-children-hbsc-study

Gire, J. T. (2002). A cross-national study of motives for drinking alcohol. Substance Use \& Misuse, 37, 215-223.

Gmel, G., Labhart, F., Fallu, J.-S., \& Kuntsche, E. (2012). The association between drinking motives and alcohol-related consequences - room for biases and measurement issues? Addiction, 107, 1580-1589.

Gmel, G., \& Rehm, J. (2004). Measuring alcohol consumption. Contemporary Drug Problems, 31, 467-540.

Graziano, F., Bina, M., \& Ciairano, S. (2010). Le funzioni del consumo di tabacco e alcolici percepite dagli adolescenti: una ricerca con il focus group. Psicologia della Salute, 3, 7-28.

Heine, S. J., \& Buchtel, E. E. (2009). Personality: The universal and the culturally specific. Annual Review of Psychology, 60, 369-394.

Hooper, D., Coughlan, J., \& Mullen, M. R. (2008). Structural equation modelling: Guidelines for determining model fit. Electronic Journal of Business Research Methods, 6, 53-60.

Kline, R. B. (Ed.). (2005). Principles and practice of structural equation modeling (2nd ed.). New York, NY: Guilford Press.

Koutakis, N., Stattin, H., \& Kerr, M. (2008). Reducing youth alcohol drinking through a parent-targeted intervention: The Orebro Prevention Program. Addiction, 103, 1629-1637.

Kuntsche, E., \& Cooper, M. L. (2010). Drinking to have fun and to get drunk: Motives as predictors of weekend drinking over and above usual drinking habits. Drug and Alcohol Dependence, 110, 259-262.

Kuntsche, E., Knibbe, R., Engels, R., \& Gmel, G. (2010a). Being drunk to have fun or to forget problems? Identifying enhancement and coping drinkers among risky drinking adolescents. European Journal of Psychological Assessment, 26, 46-54.

Kuntsche, E., Knibbe, R. A., Gmel, G., \& Engels, R. C. M. E. (2005). Why do young people drink? A review of drinking motives. Clinical Psychology Review, 25, 841-861.

Kuntsche, E., Knibbe, R. A., Gmel, G., \& Engels, R. C. M. E. (2006). Who drinks and why? A review of socio-demographic, personality, and contextual issues behind the drinking motives in young people. Addictive Behaviors, 31, 1844-1857.

Kuntsche, E., \& Kuntsche, S. (2009). Development and validation of the Drinking Motive Questionnaire Revised Short Form (DMQ-R SF). Journal of Clinical Child and Adolescent Psychology, 38, 899-908.

Kuntsche, E., \& Müller, S. (2012). Why do young people start drinking? Motives for first-time alcohol consumption and links to risky drinking in early adolescence. European Addiction Research, 18, 34-39.

Kuntsche, E., Rehm, J., \& Gmel, G. (2004). Characteristics of binge drinkers in Europe. Social Science \& Medicine, 59, 113-127.

Kuntsche, E., Stewart, S. H., \& Cooper, M. L. (2008). How stable is the motive-alcohol use link? A cross-national validation of the Drinking Motives Questionnaire Revised among adolescents from Switzerland, Canada, and the United States. Journal of Studies on Alcohol and Drugs, 69, 388-396

Kuntsche, E., Wiers, R. W., Janssen, T., \& Gmel, G. (2010b). Same wording, distinct concepts? Testing differences between expectancies and motives in a mediation model of alcohol outcomes. Experimental and Clinical Psychopharmacology, 18, 436-444.

Marsh, H. W., Hau, K.-T., \& Wen, Z. (2004). In search of golden rules:
Comment on hypothesis-testing approaches to setting cutoff values for fit indexes and dangers in overgeneralizing $\mathrm{Hu}$ and Bentler's (1999) findings. Structural Equation Modeling: A Multidisciplinary Journal, $11,320-341$.

Mazzardis, S., Vieno, A., Kuntsche, E., \& Santinello, M. (2010). Italian validation of the Drinking Motives Questionnaire Revised Short Form (DMQ-R SF). Addictive Behaviors, 35, 905-908.

Mezquita, L., Stewart, S. H., Ibáñez, M. I., Ruipérez, M. A., Villa, H., Moya, J., \& Ortet, G. (2011). Drinking motives in clinical and general populations. European Addiction Research, 17, 250-261.

Muthén, L. K., \& Muthén, B. O. (1998-2012). Mplus: User's guide (7th ed.). Los Angeles, CA: Authors

Németh, Z., Kuntsche, E., Urbán, R., Farkas, J., \& Demetrovics, Z. (2011a). Why do festival goers drink? Assessment of drinking motives using the DMQ-R SF in a recreational setting. Drug and Alcohol Review, $30,40-46$.

Németh, Z., Urbán, R., Kuntsche, E., San Pedro, E. M., Roales Nieto, J. G., Farkas, J., . . Demetrovics, Z. (2011b). Drinking motives among Spanish and Hungarian young adults: A cross-national study. Alcohol and Alcoholism, 46, 261-269.

Nunnally, J. C., \& Bernstein, I. H. (1994). Psychometric theory (3rd ed.). New York, NY: McGraw-Hill.

Perera, B., \& Torabi, M. R. (2012). Alcohol use behavior among undergraduates: A cross-cultural comparison between the United States and Sri Lanka. Southeast Asian Journal of Tropical Medicine and Public Health, 43, 1025-1034

Rice, F. P., \& Dolgin, K. G. (2002). The adolescent: Development, relationships, and culture (10th ed.). Boston, MA: Allyn and Bacon.

Roberts, B. W., \& DelVecchio, W. F. (2000). The rank-order consistency of personality traits from childhood to old age: A quantitative review of longitudinal studies. Psychological Bulletin, 126, 3-25.

Room, R. (2001). Intoxication and bad behaviour: Understanding cultural differences in the link. Social Science \& Medicine, 53, 189-198.

Schelleman-Offermans, K., Kuntsche, E., \& Knibbe, R. A. (2011). Associations between drinking motives and changes in adolescents' alcohol consumption: A full cross-lagged panel study. Addiction, 106, 1270-1278.

Steinberg, L. (2008). A social neuroscience perspective on adolescent risktaking. Developmental Review, 28, 78-106.

Stewart, S. H., Conrod, P. J., Marlatt, G. A., Comeau, M. N., Thush, C., \& Krank, M. (2005). New developments in prevention and early intervention for alcohol abuse in youths. Alcoholism: Clinical and Experimental Research, 29, 278-286.

Tabachnick, B. G., \& Fidell, L. S. (2001). Using multivariate statistics (4th ed.). Boston, MA: Allyn and Bacon.

Urbán, R., Kökönyei, G., \& Demetrovics, Z. (2008). Alcohol outcome expectancies and drinking motives mediate the association between sensation seeking and alcohol use among adolescents. Addictive Behaviors, 33, 1344-1352.

Wurdak, M., Dörfler, T., Eberhard, M., \& Wolstein, J. (2010). Tagebuchstudie zu Trinkmotiven, Affektivität und Alkoholkonsum bei Jugendlichen [Diary-based study concerning drinking motives, affectivity, and alcohol consumption among adolescents]. Sucht: Zeitschrift für Wissenschaft und Praxis/Journal of Addiction Research and Practice, $56,175-182$. 